\title{
Need To Study On a New Nontoxic Material to Relieve Cytokine Storm Caused By Viral Pandemics \\ Wansu Park*
}

Department of Pathology, College of Korean Medicine, Gachon University, Seong-nam, Republic of Korea

\begin{abstract}
The immune function is regarded to be core for protecting the host from the invasion of pathogens such as viruses, bacteria, fungi, and parasitic protozoa. Inflammation is one of major processes concerned with the immune function. But inflammation has double-sided character. Proinflammatory mediators of the uncontrolled inflammation can induce a pathologic process involved in the acute or chronic inflammation. Thus, Regulation of inflammation is becoming important more and more. Recently, Ebola and other viral diseases have been reported to cause cytokine storms (the massive overproduction of cytokines by the body's immune system), which are responsible for many of the deaths with viral pandemics. Because some non-steroidal anti-inflammatory drugs (e.g., indomethacin) show the toxicity, it is desirable to study on a new non-toxic material that can modulate the excessive increase of proinflammatory mediators (including nitric oxide, cytokine, and intracellular calcium) and consequently relieve the cytokine storm caused by viral pandemics.
\end{abstract}

Keywords: Cytokine; Cytokine storm; Inflammation; Virus; Pandemics

\section{Commentary}

The innate immune function is known to be one of the most important for protecting the host from the invasion of pathogens (e.g., viruses, bacteria, fungi, and parasitic protozoa). Inflammation is one of major processes concerned with the immune function. But inflammation has double-sided character. In detail, Gao and Hong have reported that inflammation in innate immunity is part of the nonspecific immune response that occurs in reaction to harmful stimuli such as pathologic microbes, damaged cells, irritants, or any type of bodily injury, with a primary aim of neutralizing infectious agents and initiating repair to damaged tissue, while the uncontrolled inflammatory phenomena could be a turning point for the development of acute or chronic inflammatory diseases [1]. Thus, Regulation of inflammation is becoming important more and more. Tran et al. have reported that, although inflammation is an important defense mechanism against various pathogenic invasions, the complex events and inflammatory mediators in the uncontrolled inflammation reaction can induce or sustain a pathologic process involved in different diseases, e.g., sepsis, asthma, arthritis, atherosclerosis, allergy, the metabolic syndrome, and auto-immune diseases as well as cancer [2].

Bogdan et al have reported that nitric oxide (NO), a reactive radical molecule produced from the guanidino nitrogen of L-arginine by $\mathrm{NO}$ synthase, is essential for host innate immune responses to pathogens such as bacteria, viruses, fungi, and parasites [3]. However, excessive NO production can result in the development of inflammatory diseases such as rheumatoid arthritis and autoimmune disorders [4]. Pavel et al. have reported that cytokines are pleiotropic, low-molecular-weight proteins that stimulate and regulate the immune responses to infection and inflammation by binding to cytokine receptors on the cell plasma membrane [5]. Overproduction of inflammatory cytokines is related with complications such as sepsis, lung inflammation, rheumatoid arthritis, and atherosclerosis [6].

Medina et al. have reported that macrophages play an important role in inflammatory disease through the release of factors such as NO, reactive oxygen species, cytokines (including chemokines and growth factors), and prostaglandin mediators involved in the immune response [7]. Carrithers has reported that innate immune responses mediated by mononuclear phagocytes represent the initial host response to acute viral infection and pattern recognition receptors recognize viral nucleic acid and localized injury signals to initiate proinflammatory responses and activation of adaptive immunity [8].

Pathogenic oxidative stress with infection results in macrophage reprogramming with a transient increase of intracellular calcium and this increased cytosolic calcium, in turn, results in the activation of calcium-dependent kinases, leading to enhanced proinflammatory activation [9].

Lamkanfi and Dixit have reported that the therapeutic potential of inhibiting the proinflammatory caspase-triggered pyroptosis, a proinflammatory and lytic mode of cell death, in infectious and autoimmune diseases is raised by the successful deployment of antiIL-1 therapies to control autoinflammatory diseases associated with aberrant inflammasome signaling [10]. Recently, Sordillo and Helson have reported that the terminal stage of Ebola and other viral diseases is often the onset of a cytokine storm, the massive overproduction of cytokines by the body's immune system [11]. According to report of Sordillo and Helson, the suppression of cytokine release correlates with clinical improvement in experimental models of disease conditions where a cytokine storm plays a significant role in mortality [11].

Because some non-steroidal anti-inflammatory drugs such as indomethacin show the toxicity, it is desirable to study on a new non-toxic material that can modulate the excessive increase of

*Corresponding author: Wansu Park, Department of Pathology, College of Korean Medicine, Gachon University, Seong-nam, Republic of Korea, Tel: 031750-8821; E-mail: pws98@gachon.ac.kr

Received June 30, 2015; Accepted August 01, 2015; Published August 08, 2015

Citation: Park W (2015) Need To Study On a New Nontoxic Material to Relieve Cytokine Storm Caused By Viral Pandemics. J Microb Biochem Technol 7: 242 243. doi:10.4172/1948-5948.1000216

Copyright: (c) 2015 Park W. This is an open-access article distributed under the terms of the Creative Commons Attribution License, which permits unrestricted use, distribution, and reproduction in any medium, provided the original author and source are credited. 
Citation: Park W (2015) Need To Study On a New Nontoxic Material to Relieve Cytokine Storm Caused By Viral Pandemics. J Microb Biochem Technol 7: 242-243. doi:10.4172/1948-5948.1000216

proinflammatory mediators (including NO, cytokine, and intracellular calcium) and consequently relieve the cytokine storm induced by viral infection.

\section{References}

1. Gao HM, Hong JS (2008) Why neurodegenerative diseases are progressive: Uncontrolled inflammation drives disease progression. Trends Immunol 29: 357-365.

2. Tran TV, Malainer C, Schwaiger S, Hung T, Atanasov AG, et al. (2015) Screening of Vietnamese medicinal plants for NF-Îl B signaling inhibitors: assessing the activity of flavonoids from the stem bark of Oroxylum indicum. J Ethnopharmacol 159: 36-42.

3. Bogdan C, Röllinghoff M, Diefenbach A (2000) The role of nitric oxide in innate immunity. Immunol Rev 173: 17-26.

4. O'Shea JJ, Ma A, Lipsky P (2002) Cytokines and autoimmunity. Nat Rev Immunol 2: 37-45.

5. Pavel MA, Lam C, Kashyap P, Salehi-Najafabadi Z, Singh G, et al. (2014)
Analysis of the cell surface expression of cytokine receptors using the surface protein biotinylation method. Methods Mol Biol 1172: 185-192.

6. Lin WJ, Yeh WC (2005) Implication of Toll-like receptor and tumor necrosis factor alpha signaling in septic shock. Shock 24: 206-209.

7. Medina EA, Morris IR, Berton MT (2010) Phosphatidylinositol 3-kinase activation attenuates the TLR2-mediated macrophage proinflammatory cytokine response to Francisella tularensis live vaccine strain. J Immunol 185 $7562-7572$.

8. Carrithers MD (2014) Innate immune viral recognition: Relevance to CNS infections. Handb Clin Neurol 123: 215-223.

9. Cuschieri J, Maier RV (2007) Oxidative stress, lipid rafts, and macrophage reprogramming. Antioxid Redox Signal 9: 1485-1497.

10. Lamkanfi M, Dixit VM2 (2014) Mechanisms and functions of inflammasomes. Cell 157: 1013-1022.

11. Sordillo PP, Helson L (2015) Curcumin suppression of cytokine release and cytokine storm. A potential therapy for patients with Ebola and other severe viral infections. In Vivo 29: 1-4. 